\title{
Multiparameter MRI could be used as a biomarker to guide acute treatment of stroke
}

S cans produced by two types of MRI can together be used to identify patients with acute ischemic stroke who are likely to benefit from thrombolysis, according to a large, multicenter observational study called PRE-FLAIR.

Thrombolytic drugs are the only effective treatment for acute ischemic stroke, and must be administered within $4.5 \mathrm{~h}$ of stroke onset. Late treatment is ineffective, and can be harmful beyond $6 \mathrm{~h}$ from stroke onset. Approximately $20 \%$ of strokes occur while the patient is asleep during the night, and the individual only becomes aware of their symptoms on waking. Although some of these patients will still be in the 4.5 -h window and able to benefit from thrombolysis, they are all excluded from such treatment owing to uncertainty about the time of stroke onset. "We were driven by the aim of identifying imaging surrogate markers of lesion age and tissue damage that would help to guide acute stroke treatment in the large group of patients with unknown time from symptom onset," says Götz Thomalla, who was the Principal Investigator of PRE-FLAIR.

MRI scans are used to detect brain lesions in individuals with suspected stroke. Diffusion-weighted imaging (DWI) MRI can show changes in water diffusion within $3 \mathrm{~min}$ of stroke onset, whereas fluidattenuated inversion recovery (FLAIR) MRI is sensitive to the vasogenic edema that occurs 1-4h after stroke onset. The presence of lesions on DWI but not FLAIR scans (a DWI-FLAIR mismatch) might, therefore, be used to identify patients who are within the 4.5 -h window from stroke onset and eligible for thrombolytic treatment. Previous single-center studies found some evidence to support this hypothesis, but with varying and partly conflicting results.

The PRE-FLAIR study involved individual data sets from eight stroke centers and two stroke studies, producing
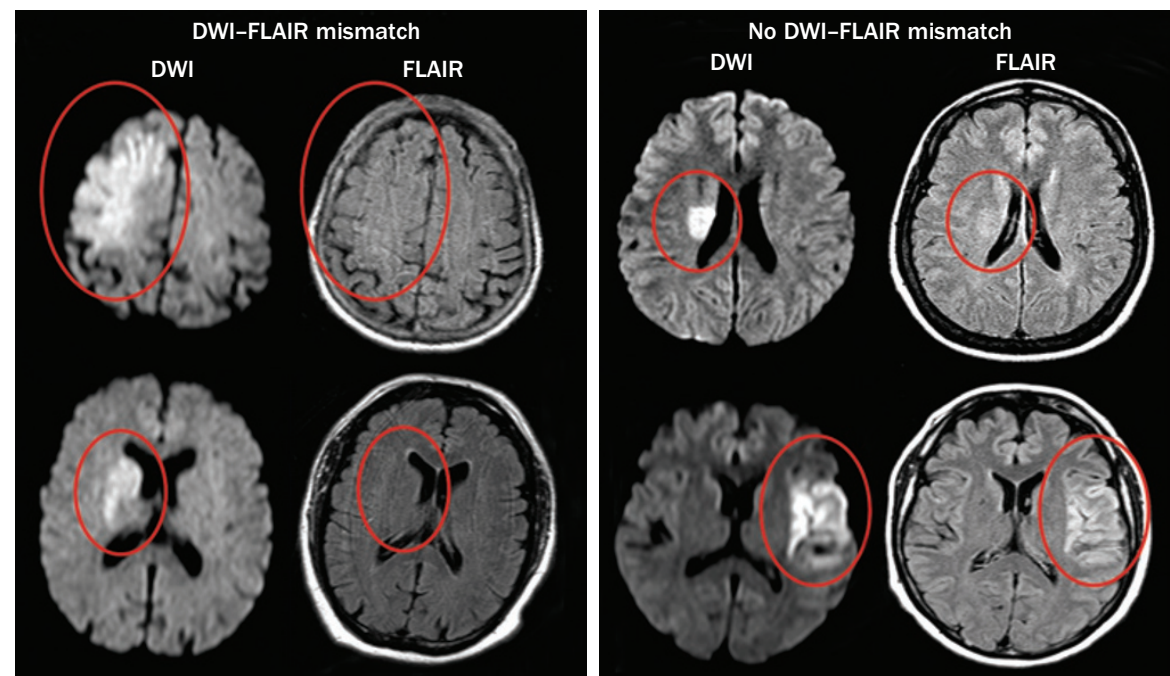

Time from stroke onset is likely to be $<4.5 \mathrm{~h}$ in patients with mismatched DWI and FLAIR MRI scans (left) and $>4.5 \mathrm{~h}$ in patients with visible lesions in both DWI and FLAIR MRI scans (right). Image courtesy of G. Thomalla.

a cohort of 543 patients. All patients had a known time of stroke onset, and DWI and FLAIR MRI scans taken within $12 \mathrm{~h}$ of symptom onset. The primary outcome measure was the specificity and positive predictive value of DWI-FLAIR mismatch in the identification of patients with acute stroke who were within the recommended 4.5-h time window for thrombolysis with intravenous alteplase. Two neurologists, who were blinded to the patients' time of stroke onset, analyzed the MRI scans. A DWI-FLAIR mismatch was defined as the presence of an ischemic lesion on the DWI scan in the absence of a parenchymal hyperintensity in the corresponding brain region on the FLAIR scan. "We wanted to keep the image analysis process as simple as possible, so that it can be easily transferred into clinical practice," explains Thomalla.

Of the 245 patients in the study who had a DWI-FLAIR mismatch, 204 were within the 4.5 -h time window from stroke onset, representing a $78 \%$ specificity and $83 \%$ positive predictive value for this imaging biomarker; such low false-positive rates are particularly important given that late treatment with thrombolytics can worsen symptoms. Conversely, 125 of the 271 patients who showed lesions on both DWI and FLAIR scans were seen within $4.5 \mathrm{~h}$ of stroke onset and were, therefore, eligible for thrombolysis. This modest sensitivity and negative predictive value of the DWIFLAIR mismatch suggests the need to test additional MRI indices.

"The methodology in this study was sound," says Ramon Gilberto Gonzalez, who is Director of the Neuroradiology Division of Massachusetts General Hospital, and was not involved in the study. "It is possible -and even reasonable to say - that MRI is superior to time as a surrogate for the physiological abnormalities in the brain of the stroke patient."

"Our study paves the way for the use of DWI-FLAIR mismatch as a criterion to enrol patients with 'wake-up stroke' into a randomized clinical trial, which we are currently organizing and will start by the end of 2012," concludes Thomalla.

Katie Kingwell 